\title{
Optimization of static traffic allocation policies
}

\author{
M.B. Combé* and O.J. Boxma** \\ CWI, P.O. Box 94079, 1090 GB Amsterdam, The Netherlands
}

\begin{abstract}
Combé, M.B., O.J. Boxma, Optimization of static traffic allocation policies, Theoretical Computer Science 125 (1994) 17-43.

We consider the traffic allocation problem: arriving customers have to be assigned to one of a group of servers. The aim is to optimize system performance measures, such as mean waiting time of a customer or total number of customers in the system, under a given static allocation policy. Two static policies are considered: probabilistic assignment and allocation according to a fixed pattern. For these two policies, general properties as well as optimization aspects are discussed.
\end{abstract}

\section{Introduction}

In a distributed computer system, tasks generated by a group of users can be distributed over a number of available processors. This contrasts with systems in which a single processor provides (global) computer capacity for all users, or systems in which each user is provided with its own local processor, usually with very limited capacity.

An operational aspect of such a distributed system is the availability of a load balancing protocol. Such a protocol balances the work-load over the servers, aiming to optimize performance measures for the system, such as mean amount of work-load, throughput, or mean waiting times of jobs.

Load balancing is required in many situations where a work-load is offered to a number of servers with limited capacity. Apart from distributed systems, one may e.g. think of the transmission of messages along one of several available paths of a communication network.

Correspondence to: M.B. Combé, CWI, P.O. Box 94079, 1090 GB Amsterdam, The Netherlands.

* Supported by NFI.

** Part of the research was supported by the European grant BRA-QMIPS of CEC DG XIII. 
An important element of a load balancing protocol is the information it requires to operate. This information can range from total knowledge about the system at any point in time, to only information about some basic characteristics, like arrival rate and service times. In general, the term dynamic is used for policies which operate under time-dependent information, whereas protocols operating under time-independent characteristics of the system are called static.

It is clear that the more information is available for making decisions, the better the allocation of work-load can be. Dynamic policies in general perform better than static policies. However, static load balancing protocols are also of considerable interest. First of all, the situation of total knowledge at all times is unrealistic. From a viewpoint of costs, overhead grows as the amount of information to be exchanged, stored and processed increases. Moreover, dynamic policies are not always that effective: there will always be some kind of time delay between updates of the system's current state, and this time delay can have a considerable effect on the quality of the protocol.

A second reason for studying static allocation policies is that they can be useful tools in the design phase of a computer- or communication network. Static policies can provide performance bounds for dynamically controlled systems; the performance measures under static policies are in general evaluated reasonably quickly, whereas dynamic policies are harder to analyse and their performance can only be evaluated with time consuming methods.

In this paper we consider the traffic allocation problem for two static allocation protocols for the model of a single Poisson stream of jobs offered to a fixed number of server stations. The allocation protocols we study are static in the sense described above; only the traffic intensity and the server characteristics are used. We give an overview of the results for these policies and also extend optimization procedures for some models.

In the remainder of this section a brief survey of related literature and an outline of the paper are given.

\subsection{Related literature}

Several papers have addressed the load balancing problem. Below, we refer to two overview papers for the general load balancing problem, before giving a more extensive overview of the traffic allocation problem. Wang and Morris [27] give a taxonomy for the current load balancing protocols. They formulate the load balancing problem in its most general form, also discriminating between server initiative protocols, i.e. the servers determine from which input sources they draw their customers, and source initiative protocols, i.e. at the moment of arrival in the system jobs are (irrevocably) routed to one of the servers. Wang and Morris [27] provide numerical comparisons, based on analysis and simulation, of various allocation protocols. An overview of load balancing policies and their performances is also given by Boel and van Schuppen [4]. They consider the problem from a control point of view and discuss the question as to what amount of information is required at the 
routing points to achieve good system performance. Their paper concentrates on analytically and numerically tractable models.

Two static allocation policies have been proposed for the traffic allocation problem: viz. probabilistic assignment and pattern allocation. With the probabilistic policy, each arriving customer is routed to one of the servers with fixed probabilities. Under pattern allocation, each arriving customer is routed to a server according to an allocation table.

For probabilistic allocation, Buzen and Chen [8] present an algorithm for determining the allocation which minimizes the mean sojourn time of a customer. Their mathematical programming formulation can easily be extended for various other performance measures and fits into the framework of Ibaraki and Katoh [13] for resource allocation problems (RAP). Optimal probabilistic load balancing has been studied by Jean-Marie [15] for the case of two parallel exponential servers and resequencing.

Numerical comparisons (cf. [27]) reveal that dynamic allocation policies lead to considerably better results than probabilistic allocation. Yum [28] proposed the pattern allocation policy ("semi-dynamic deterministic routing"), which performs notably better than the probabilistic allocation policy (cf. [1,28]). The reason for this is that the arrival processes at the servers under the pattern allocation policy are less irregular than under probabilistic allocation. However, constructing the optimal allocation pattern is an unsolved problem as yet. For the case of two identical exponential servers, Ephremides et al. [11] proved that alternatively assigning customers to each queue is optimal, a result which was extended by Ramakrishnan [21] for the model with more than two identical exponential servers. Ramakrishnan [21] also proposed a useful approximation procedure for the case of nonidentical exponential servers.

The present paper extends the approximation procedure proposed by Ramakrishnan [21] in several directions, in particular allowing general service time distributions. We also give an overview of the results for the two above-mentioned static allocation policies. By comparing both policies from a more theoretical viewpoint than in most previous studies, we develop insights into general allocation problems and clarify some reported, but hitherto unexplained, properties.

\subsection{Outline of the paper}

In Section 2 a mathematical description of the allocation problem is presented, and the probabilistic allocation policy is discussed. In Section 3 we argue that allocation policies which result in more regular arrival processes than the Poisson arrival process are to be preferred to probabilistic allocation. There also the pattern allocation policy is introduced. In Section 4 an optimization procedure for pattern allocation is presented. In Section 5 the performance measures under both allocation policies are numerically compared for various models. We also compare both policies with a dynamic policy that is expected to outperform most policies for the objective 
functions we consider. Section 6 discusses three extensions of the basic traffic allocation problem. The first extension deals with the case of a general arrival process. The second model describes the case in which all server stations receive a ("dedicated") Poisson arrival stream, on top of which an extra arrival stream has to be allocated. The third extension considers allocation to multiple server stations.

\section{Probabilistic allocation}

Before studying the probabilistic allocation policy, we first present a mathematical description of the traffic allocation problem.

\subsection{Model description}

Customers arrive at a routing point according to a Poisson process with rate $\Lambda$. At the instance of arrival, a customer has to be assigned to one of $N$ single servers in parallel. This assignment is irrevocable.

The service time $\boldsymbol{B}_{i}$ of a customer that is assigned to server $i$ has general distribution $B_{i}(\cdot)$, with first and second moment $\beta_{i}$ and $\beta_{i}^{(2)}$, respectively. All service times are independent.

Let $P$ denote an allocation policy and $p_{i}, i=1, \ldots, N$, be the fraction of the customers that is routed to server $i$ under policy $P$.

In our traffic allocation problem, the aim is to minimize

$$
\sum_{i=1}^{N} f_{i}(P) C_{i} E W_{i}(P)
$$

In (2.1) $E W_{i}(P)$ denotes the mean waiting time of a customer assigned to server $i$ under allocation policy $P . C_{i}$ is the cost associated with waiting one time unit at queue $i$. The factors $f_{i}(P)$ are additional, load-dependent, weight factors. The objective function can have various interpretations by varying $f_{i}(\cdot)$ and $C_{i}$. For example, if $f_{i}(P)=p_{i}$ and $C_{i}=1, i=1, \ldots, N$, then the objective function represents the mean waiting time of an arbitrary customer. Or, with $f_{i}(P)=\Lambda p_{i}$ and $C_{i}=\beta_{i}$, Little's law shows that the objective is to minimize the mean total amount of work in the queues. Instead of $E W_{i}(P)$, also $E \boldsymbol{R}_{i}(P)$, the mean sojourn time of a customer assigned to queue $i$, could have been used in (2.1).

\subsection{Probabilistic allocation}

As described in the introduction, the assignment of an arriving customer to a queue can depend on all kinds of information contained in the history and the present state of the system. In this section we discuss the probabilistic allocation policy, also known as random splitting. Under this policy, a fraction $p_{i}$ of the arrivals is routed to queue $i$ by assigning a customer, arriving at the routing point, to server station $i$ with 
probability $p_{i}, i=1, \ldots, N\left(\sum_{i} p_{i}=1\right)$. These probabilities $p_{i}$ are the same for all customers, and do not change in time. Let $P_{\mathrm{pr}}$ denote the class of probabilistic allocation policies. This class can be completely described by $P_{\mathrm{pr}}=\left\{p \mid p \in[0,1]^{N}\right.$, $\left.\sum_{i=1}^{N} p_{i}=1\right\}$.

The probabilistic allocation policy is static in the sense that when a customer has to be routed to one of the queues, no information about the history and the present state of the system is used. Under $P \in P_{\mathrm{pr}}$, the arrival process at queue $i$ is Poisson with intensity $\lambda_{i}=p_{i} \Lambda, i=1, \ldots, N$, and the objective function becomes

$$
\sum_{i=1}^{N} f_{i}(P) C_{i} E W_{i}(P)=\sum_{i=1}^{N} f_{i}(P) C_{i} \frac{p_{i} \Lambda \beta_{i}^{(2)}}{2\left(1-p_{i} \Lambda \beta_{i}\right)} .
$$

Among the first to study the probabilistic allocation policy were Buzen and Chen [8]. Their aim was to minimize the mean sojourn time of a customer for a model with generally distributed service times at the server stations. They solved the problem using standard mathematical programming techniques.

As an example, we take $f_{i}(P)=\lambda_{i} / \Lambda$ in (2.2) and solve the allocation problem. In this case, the objective is to minimize the mean weighted waiting time of a customer or, using Little's law, to minimize a weighted sum of the mean number of waiting customers in the system. To obtain the assignment probabilities $p_{i}^{*}=\lambda_{i}^{*} / \Lambda$ which minimize this function, the following mathematical programming problem (MPP) has to be solved

PA1:

$$
\begin{aligned}
& \min \sum_{i=1}^{N} \lambda_{i} C_{i} \frac{\lambda_{i} \beta_{i}^{(2)}}{2\left(1-\lambda_{i} \beta_{i}\right)} \\
& \text { s.t. } \sum_{i=1}^{N} \lambda_{i}=\Lambda, \\
& 0 \leqslant \lambda_{i}<\frac{1}{\beta_{i}}, \quad i=1, \ldots, N .
\end{aligned}
$$

Note that the objective function in PA1 can be separated in terms

$$
T_{i}=\lambda_{i} C_{i} \frac{\lambda_{i} \beta_{i}^{(2)}}{2\left(1-\lambda_{i} \beta_{i}\right)},
$$

which are strictly convex functions in $\lambda_{i}$. It can also be verified that PA1 has a feasible solution provided that $\sum_{i} 1 / \beta_{i}>\Lambda$, i.e. the arrival rate does not exceed the total service capacity. Here and in the remainder of the paper it is assumed that such is the case.

Problem PA1 allows an analytical solution. To find this solution, we first relax PA1 by dropping the last constraint. Using the standard Lagrange-multiplier techniques 
we obtain, with $\delta$ the Lagrange-multiplier, the following first-order Kuhn-Tucker constraints:

$$
\begin{aligned}
& \frac{\mathrm{d}}{\mathrm{d} \lambda_{i}}\left\{\lambda_{i} C_{i} \frac{\lambda_{i} \beta_{i}^{(2)}}{2\left(1-\lambda_{i} \beta_{i}\right)}\right\}=\delta, \quad i=1, \ldots, N, \\
& \sum_{i=1}^{N} \lambda_{i}-\Lambda=0 .
\end{aligned}
$$

From (2.6) and remarking that (2.3) concerns a sum of terms $T_{i}$ which are convex functions of $\lambda_{i}$ we find the unique optimal values $\lambda_{i}^{*}$

$$
\lambda_{i}^{*}=\frac{1}{\beta_{i}}-\frac{1}{\beta_{i}}\left(\sqrt{1+\frac{2 \beta_{i} \delta}{C_{i} \beta_{i}^{(2)}}}\right)^{-1}, \quad i=1, \ldots, N,
$$

in which the value of the Lagrange-multiplier $\delta$ is determined by the constraint (2.7). The optimal splitting probabilities are given by $p_{i}^{*}=\lambda_{i}^{*} / \Lambda, i=1, \ldots, N$. In (2.8) we see that $0 \leqslant \lambda_{i}^{*}<1 / \beta_{i}, i=1, \ldots, N$; so the vector $\lambda^{*}$ is also the solution of PA1.

This example shows us the structure of our traffic allocation problem. The objective function is separable in (strictly convex) terms $T_{i}$, each term $T_{i}$ being a function of $\lambda_{i}$. Hence the solution of PA1 is determined by the derivatives of the terms (cf. (2.6)) rather than their values.

A second observation follows from (2.7) and (2.8): if $\Lambda>0$, then $\lambda_{i}^{*}>0$ for all $i$. This is a direct consequence of the above-mentioned properties; in the example we have $\mathrm{d} T_{i} /\left.\mathrm{d} \lambda_{i}\right|_{\lambda_{i}=0}=0$ and $\mathrm{d} T_{i} / \mathrm{d} \lambda_{i}$ is an increasing function in $\lambda_{i}$, hence $\lambda_{i}^{*}>0$, provided that $\Lambda>0$.

However, for other naturally arising objective functions, such as $\sum_{i} C_{i} E W_{i}$ or $\sum_{i} f_{i} C_{i} E \boldsymbol{R}_{i}$, with $\boldsymbol{R}_{i}$ denoting the sojourn time of a customer routed to queue $i$, the optimal values of some $\lambda_{i}$ 's (and $p_{i}$ 's) may be equal to zero. For these objective functions $\mathrm{d} T_{i} /\left.\mathrm{d} \lambda_{i}\right|_{\lambda_{i}=0}$ can be so large, relative to the other queues, that it is advantageous not to assign customers to queue $i$, but to allocate all arrivals amongst the other queues.

For the latter objective functions the MPPs have the same structure as PA1. Usually, these MPPs do not allow an analytical solution. However, PA1 can be solved quite easily numerically, due to its special structure: the control variables only interact through the linear restriction (2.4). This characteristic is typical for the class of resource allocation problems (RAP), as studied by Ibaraki and Katoh [13]. In their book they also consider a RAP which has almost the same form as PA1, the only difference being that the control variables are allowed to equal the upper bounds.

In [10] we present an algorithm to solve the traffic allocation problem that has a separable objective function, consisting of strictly convex terms, and that has strict upper bounds on the control variables. The algorithm is a variant of the procedure RANK in $\left(\left[13\right.\right.$, p. 19]). The algorithm first determines the set of queues for which $\lambda_{i}^{*}>0$; 
for that set it subsequently solves a set of equations of the form of (2.6) and (2.7). The algorithm strongly depends on the strict convexity of the terms. Due to this property, there is only one local minimum, which consequently has to be the optimal solution for the allocation problem. If the objective function is not separable into strictly convex terms, then in general there may exist several local minima for the allocation problem. Moreover, in most situations only approximately optimal allocation probabilities can be obtained. One of the cases in which the property of strictly convex terms may not hold is the traffic allocation problem with a general arrival process, as studied in Tang and van Vliet [26]. Their method involves an algorithm for quadratic programming and provides one of the local minima. They also argue that this local minimum should be close to the global minimum.

\section{Less variable arrival processes}

Intuitively, one expects that when traffic allocation leads to a more regular arrival process, then the mean waiting times are reduced and consequently also the value of the objective function. However, it is very difficult to prove such statements, except for special cases. A detailed investigation of these issues would not fit into the framework of this paper, and hence we restrict ourselves to presenting some basic results on comparison between queueing systems, along with a special case to support the above-mentioned intuition.

In this section we discuss the single server queue with an arrival process that is more regular than the Poisson process, and we argue that for an important class of such arrival processes, the behaviour of the mean waiting time as a function of the load is better than for Poisson arrivals.

General comparisons of GI/G/1 systems are presented by Stoyan [25]. Particularly useful for our purposes is his Theorem 5.2.1, which states the following monotonicity property for the waiting times.

Lemma 3.1. Consider two $G I / G / I$ queueing systems with identically distributed service times. If for the interarrival times $A_{1}$ and $A_{2}, A_{1} \leqslant_{\mathrm{c}} A_{2}$, then also for the steady-state waiting times $W_{1} \leqslant_{\mathrm{c}} W_{2}$.

Here $\leqslant_{\mathrm{c}}$ denotes the convex stochastic ordering for random variables, and indices 1,2 refer to the two queueing systems. Since $W_{1}$ and $W_{2}$ are positive random variables, $\boldsymbol{W}_{1} \leqslant{ }_{\mathrm{c}} \boldsymbol{W}_{2}$ implies $E \boldsymbol{W}_{1}^{r} \leqslant E \boldsymbol{W}_{2}^{r}, r=1,2, \ldots$

In particular, if $E A_{1}=E A_{2}, A_{1} \leqslant_{c} A_{2}$ holds in the following two cases:

(i) $\boldsymbol{A}_{1}$ is constant (cf. [25, Example 1.9(a)],

(ii) $A_{1}$ is NBUE and $A_{2}$ has an exponential distribution.

A stochastic variable $X$ with distribution function $F$ is "new better than used in expectation" (NBUE) if $\int_{t}^{\infty}(1-F(x)) \mathrm{d} x /(1-F(t)) \leqslant E X$ for all $t \geqslant 0$. Note that if $X$ has 
an increasing failure rate, $\boldsymbol{X}$ is NBUE. As examples, $\operatorname{Gamma}(\Lambda, y)$ with $y \geqslant 1$, Weibull $(\Lambda, y)$ with $y \geqslant 1$ and uniformly distributed random variables are NBUE (cf. $[25$, Ch. 1]). The Gamma $(\Lambda, y)$ case is now discussed in more detail, as it plays an important role in the remainder of the paper.

$\operatorname{Gamma}(\Lambda, y)$ : Consider a $\operatorname{Gamma}(\Lambda, y) / \mathrm{M} / 1$ queueing model with $y>1$, so that the arrival process has a coefficient of variation which is smaller than that of a Poisson process. Let $\mu$ be the service rate and $\Lambda / \mu y<1$, i.e. the queue is stable. In this queue the mean waiting time of a customer $E W^{G}$ is given by $\omega /(\mu(1-\omega))$, with $\omega=\operatorname{Pr}\left\{W^{G}>0\right\}$ the smallest positive real solution of $x=\alpha(\mu(1-x)), \alpha(\cdot)$ being the Laplace-Stieltjes transform of the arrival process (cf. [9]). For Gamma $(\Lambda, y)$, we have $\alpha(x)=(\Lambda /(\Lambda+x))^{y}$.

Firstly, for this queueing model $E W^{G}<E W^{M}$, which follows from Lemma 3.1. Here $E W^{\mathrm{M}}$ denotes the mean waiting time in the $\mathrm{M} / \mathrm{M} / 1$ queue with arrival rate $\Lambda / y$ and service rate $\mu$. Secondly, it is readily verified that

$$
\frac{\mathrm{d}}{\mathrm{d} \Lambda} E W^{\mathrm{G}} \downarrow 0 \text { as } \Lambda \downarrow 0 .
$$

The consequence of these two properties for the model with $N \operatorname{Gamma}\left(\lambda_{i}, y_{i}\right) / \mathrm{M} / 1$ queues, $y_{i}>1, i=1, \ldots, N$ is: if one has to assign intensities $\lambda_{i}$ such that the overall arrival rate $\sum_{i} \lambda_{i} / y_{i}=\Lambda$ while the objective is to minimize $\sum_{i} E W_{i}^{\mathrm{G}}$, then $\lambda_{i}>0$ for all $i$. Moreover, the value of the objective function will be lower than if a Poisson $\Lambda$ arrival stream had been allocated probabilistically to the $N$ stations.

For the $\operatorname{Gamma}(\Lambda, y) / \mathrm{M} / 1$ queueing model, we also find that

$$
\frac{\mathrm{d}}{\mathrm{d} y} E W^{\mathrm{G}} \downarrow 0 \text { as } y \rightarrow \infty .
$$

As a consequence, suppose that for the model with $N \operatorname{Gamma}\left(\Lambda, y_{i}\right) / \mathrm{M} / 1$ queues one has to assign $y_{i}$ 's such that $\sum_{i} 1 / y_{i}=1$, i.e. the sum of the arrival rates at the queues is $\Lambda$. Then $1 / y_{i}>0$ for all $i$. This special queueing model is used in the next section as an approximation for a queue with a special nonrenewal arrival process.

\subsection{Pattern allocation - the $M A P / G / 1$ queue}

Next we introduce a traffic allocation policy which allocates the Poisson arrival stream such that the arrival processes at the queues are less variable than under probabilistic assignment, but which still is static in the sense that no state information of the queues is used and that the allocations are time independent. This policy is pattern allocation. Pattern allocation uses an infinite string of integers $\left\{a_{1}, a_{2}, \ldots\right.$, $\left.a_{n-1}, a_{n}, a_{n+1}, \ldots\right\}$, where $a_{n}$ denotes the number of the queue to which the $n$th customer in the arrival process is routed. For practical reasons, it is assumed that this string contains a sub-pattern $S$ of finite length $M$ which is repeated over and over. Thus $a_{i}=a_{i+k M}$ for all $i=1, \ldots, M$ and $k=1,2, \ldots$. Like for the probabilistic allocation 
policy we can completely describe $P_{\mathrm{pa}}$, the class of pattern allocations. This is done by $P_{\mathrm{pa}}=\left\{a \mid a \in[1, \ldots, N]^{k}, k=1,2, \ldots\right\}$.

Let $A_{i_{n}}$ be the time between the $n$th and $(n+1)$ th arrival at queue $i$. Under pattern allocation, the distributions of $A_{i_{n}}$ form a repeated sequence of Erlang distributions. For example, if $S=\{1,2,1,3,4,1,2\}$, then the sequence of the interarrival distributions at queue 1 is a repetition of $\{\operatorname{Erlang}(\Lambda, 2)$, $\operatorname{Erlang}(\Lambda, 3)$, $\operatorname{Erlang}(\Lambda, 2)\}$.

The pattern allocation policy was first introduced by Yum [28] as semi-dynamic deterministic routing. For the cases of two and infinitely many identical exponential server stations, Yum [28] shows a considerable reduction in mean waiting time if the pattern allocation policy is used instead of probabilistic allocation.

The arrival processes which result from pattern allocation fall into the class of the Markovian arrival process (MAP). The MAP studied by us is characterized by a continuous-time Markov process with finite state space $\{1, \ldots, M\}$, where arrivals can occur only at transition epochs in the Markov process. The transitions at which an arrival takes place are defined by a $0-1 M \times M$ matrix $D$, with $D_{i j}=1$ if and only if the transition from $i$ to $j$ in the Markov process is associated with an arrival. In the MAP arising under pattern allocation, the Markov chain has the special property that only transitions from state $i$ to state $(i \bmod M)+1$ can occur. In Appendix A we present some results from [17] for the MAP/G/1 queue, whose analysis is based on the matrix geometric techniques as developed by Neuts [19] and Ramaswami [22]. Using more classical techniques, Agrawala and Tripathi [2] analyse the waiting times in the MAP/M/1 queue for the typical MAP that we consider.

Observe that the MAP in general is not a renewal process. The earlier mentioned comparisons from [25] are for GI/G/1 queues and do not apply to MAP/G/1 queues. Besides, a useful characterization of the irregularity of a MAP is much more complicated than for GI arrival processes. However, in order to compare the MAP/G/1 queue with an $\mathrm{M} / \mathrm{G} / 1$ queue with the same service time distribution we state the following conjecture, which is based on the observations made earlier in this section and supported by numerical experience.

Conjecture 3.2. Consider a stable $M / G / 1$ queue in which the arrival rate is $p \Lambda$, with $p<1$, and the service time has distribution $B(\cdot)$. Then there exists a MAP with transition rate $\Lambda$ in all states of the underlying Markov chain, and overall arrival rate closely approximating $p \Lambda$ from above, such that in the $M A P / G / I$ queue with the same service time distribution $B(\cdot), E W^{\mathrm{MAP}}<E W^{\mathrm{M}}$, where $\boldsymbol{W}^{\mathrm{MAP}}$ and $\boldsymbol{W}^{\mathrm{M}}$ denote the steady-state waiting times of customers in the $M A P / G / 1$ queue and $M / G / 1$ queue, respectively.

Conjecture 3.2 is clarified by viewing the Poisson $(p \Lambda)$ arrival process as the result of a probabilistic allocation and the MAP as the result of a pattern allocation. Let $M$ be the number of phases in the MAP. Then in the pattern allocation out of every $M$ arriving customers an exact fraction $p$ is routed to the queue, whereas under probabilistic allocation, this fraction is equal to $p$ only in expectation. Moreover, in 
the MAP the arrivals can be better regulated, e.g. for $p=\frac{2}{5}$ every second and fifth customer can be routed to the queue.

Note that not for all MAPs with phase intensity $\Lambda$ and overall arrival rate $p \Lambda$, $E W^{\mathrm{MAP}}<E W^{\mathrm{M}}$; for example, again viewing the MAP as the result of pattern allocation, when of every $2 M$ customers the first $M$ are routed to the queue, then for $\frac{1}{2}<\Lambda \beta-1$, the mean waiting time of a customer at the queue tends to infinity as $M \rightarrow \infty$, while $p=\frac{1}{2}$ and $E W^{\mathrm{M}}<\infty$.

Also note that the refinement "closely approximating $p \Lambda$ from above" has to be made, because for $p$ irrational there does not exist a MAP with finite state space of the underlying Markov process such that the overall arrival rate is exactly equal to $p \Lambda$.

A benefit of the pattern allocation policy, besides lowering the value of the objective function, is that it is more robust than probabilistic allocation. For example, from the explicit expression for the mean waiting times in an exponential server queue (cf. $\operatorname{Gamma}(\Lambda, y)$ case), it follows that slightly altering the arrival intensity in an Erlang $(\Lambda, 2) / \mathrm{M} / 1$ queue has less influence than changing the arrival intensity in an $\mathrm{M} / \mathrm{M} / 1$ queue.

In this section we have argued that in the traffic allocation problem the pattern allocation policy is to be preferred to the probabilistic allocation policy, because of the reduction of variability in the arrival processes. In the next section we turn our attention to an optimization procedure for the pattern allocation policy.

\section{Optimal pattern allocation}

The mean waiting time of a customer in the MAP/G/1 queue is given by formula (A.5) of Appendix A; it is a closed expression which can be evaluated. However, (A.5) is not very suitable for a direct optimization procedure; the matrix structure of (A.5) makes an exact analytical optimization actually impossible. This contrasts with probabilistic allocation where only the $N$ optimal assignment probabilities $p_{i}^{*}$ have to be determined and where the simple structure of the objective function (2.3) allows an analytical solution of the MPP PA1.

Moreover, for pattern allocation it is impossible to determine the optimal allocation pattern by comparing patterns; there are too many patterns with length smaller than some practical bound, and the matrix operations involved in the evaluation of expression (A.5) are too time consuming.

We therefore have to resort to an approximate optimization procedure. Our procedure consists of two steps:

(1) Approximate $p_{i}^{*}, i=1, \ldots, N$, the queue assignment frequencies in the optimal allocation pattern.

(2) Use these frequencies for the construction of the allocation pattern.

In this section our attention is mainly devoted to step (1). The problems related to step (2) are more of a combinatorial nature, and in fact a quite difficult 
cyclic scheduling problem has to be solved. In Remark 4.3 we mention some of the difficulties occurring here, and in Appendix B we present a heuristic for building an allocation pattern from a set of allocation frequencies.

Due to the matrix operations involved, comparing assignment frequencies directly using (A.5) is too time consuming, so further approximations have to be made. To avoid the matrix operations we approximate the MAP with a GI arrival process.

An obvious option for this GI arrival process is the Poisson arrival process. In step (1), the fractions $p_{i}^{*}, i=1, \ldots, N$ are then approximated by the optimal probabilistic allocation. However, in general this does not lead to the optimal allocation pattern, as illustrated in [1] for the traffic allocation problem with the mean sojourn time of a customer as objective function.

A good choice for a GI approximation of the MAP is the Gamma arrival process, an arrival process with Gamma distributed interarrival times. The first step then is to determine the optimal allocation fractions for a model in which we are to assign customers from an infinite reservoir of customers to $N$ parallel Gamma/G/1 queues maintaining an overall arrival rate $\Lambda$. This we call the Gamma approximation procedure.

The idea of approximating the arrival process with a Gamma arrival process was first used by Ramakrishnan [21], who studied various allocation policies for the case of exponentially distributed service times. Using the exact expression for the mean waiting times in the Gamma/M/1 queue (cf. case 3.1 of Section 3), Ramakrishnan numerically solved the Gamma/M/1 allocation problem for the case of two queues.

The $\operatorname{Gamma}(\Lambda, y)$ arrival process appears to be a reasonable approximation for the MAP with overall arrival intensity $\Lambda / y$. It possesses the same phase character as the MAP, and if $y$ is an integer and the MAP is as regular as possible, both arrival processes have the same Erlang interarrival times.

The Gamma arrival process can be viewed as the ideal MAP; if from an infinite reservoir of customers $a_{i}$ customers out of every $M$ have to be routed to queue $i$ such that the interarrival times of the customers are i.i.d. and the sum of $a_{i}$ interarrival times has an $\operatorname{Erlang}(\Lambda, M)$ distribution (the length of the arrival pattern), then the interarrival time of a customer has a $\operatorname{Gamma}\left(\Lambda, M / a_{i}\right)$ distribution. This implies that a $\operatorname{Gamma}\left(\Lambda, M / a_{i}\right)$ arrival process is more regular than the MAP with the same arrival intensity. Hence we expect the mean waiting times in the MAP/G/1 queue to be bounded from below by the mean waiting times in the corresponding Gamma/G/1 queue. Again, such a statement is hard to prove, except for the case of exponential servers, for which the proof readily follows from the results in [12].

Unfortunately, the expression for the mean waiting times of customers in a Gamma/G/1 queue (cf. [9]) is too complicated to be useful in an optimization procedure, and hence we have to resort to more simple approximate expressions for this mean waiting time.

The next part of this section is devoted to the actual determination of the allocation fractions. For the mean waiting times in a Gamma/G/1 queue we apply the 
two-moment approximation proposed by Krämer and Langenbach-Belz (KLB; cf. [16]) for $\mathrm{Gl} / \mathrm{G} / 1$ queues:

$$
E W=\frac{\rho \beta}{2(1-\rho)}\left[c_{\mathrm{a}}^{2}+c_{\mathrm{s}}^{2}\right] \exp \left\{-\frac{2(1-\rho)}{3 \rho} \frac{\left(1-c_{\mathrm{a}}^{2}\right)^{2}}{c_{\mathrm{a}}^{2}+c_{\mathrm{s}}^{2}}\right\}
$$

in which $\beta$ is the mean service time, $\rho$ is the load of the queue, and $c_{\mathrm{a}}^{2}$ and $c_{\mathrm{s}}^{2}$ denote the squared coefficient of variation (variance divided by squared mean) of the arrival time and service time distributions, respectively. Obviously (4.1) is exact if the arrival process is Poisson.

A number of approximations for the mean waiting time in the GI/G/1 queue are compared in Shanthikumar and Buzacott [24]. From [24] the Marshall approximation appears to be a good alternative for the KLB approximation.

For a $\operatorname{Gamma}(\Lambda, y)$ process, the arrival rate $\lambda$ is given by $\Lambda / y$ and $c_{\mathrm{a}}^{2}=1 / y$, and for the Gamma/G/1 queue, (4.1) thus becomes

$$
E W=\frac{\Lambda \beta^{2}}{2(y-\Lambda \beta)}\left[\frac{1}{y}+\frac{\beta^{(2)}-\beta^{2}}{\beta^{2}}\right] \exp \left\{-\frac{2(y-\Lambda \beta)}{3 \Lambda \beta} \frac{(1-1 / y)^{2}}{1 / y-1+\beta^{(2)} / \beta^{2}}\right\} .
$$

With (4.2) we can formulate the MPP for the Gamma approximation procedure. For objective function (2.2), substituting $\alpha_{i}:=f_{i}=\lambda_{i} / \Lambda=1 / y_{i}$ we find

GA1:

$$
\begin{aligned}
& \min \sum_{i=1}^{N} \frac{\Lambda \alpha_{i}^{2} \beta_{i}^{2} C_{i}}{2\left(1-\alpha_{i} \Lambda \beta_{i}\right)}\left[\alpha_{i}+\frac{\beta_{i}^{(2)}-\beta_{i}^{2}}{\beta_{i}^{2}}\right] \exp \left\{-\frac{2\left(1-\alpha_{i} \Lambda \beta_{i}\right)}{3 \alpha_{i} \Lambda \beta_{i}} \frac{\left(1-\alpha_{i}\right)^{2}}{\alpha_{i}-1+\left(\beta_{i}^{(2)} / \beta_{i}^{2}\right)}\right\} \\
& \text { s.t. } \sum_{i=1}^{N} \alpha_{i}=1, \\
& 0 \leqslant \alpha_{i}<\frac{1}{\Lambda \beta_{i}}, \quad i=1, \ldots, N .
\end{aligned}
$$

Problem GA1 has the same structure as PA1 in Section 2, and hence it can easily be solved numerically with the algorithm presented in [10]. Note that

$$
\left.\lim _{\{\varepsilon \downarrow 0\}} \frac{\mathrm{d} E W_{i}}{\mathrm{~d} \alpha_{i}}\right|_{\alpha_{i}=\varepsilon}=0 .
$$

Hence not only the optimal assignment frequencies resulting from $\mathrm{GA} 1$ are all greater than 0 , but this would also be the case for the objective function $\sum_{i} C_{i} E W_{i}$. The latter was not always the case for the optimal probabilistic allocation.

Earlier in this section we stated that the mean waiting times in the MAP/G/1 queue are bounded from below by the mean waiting times in the corresponding Gamma/G/1 queue. Consequently, the solution of GA1 provides an approximate lower bound for the mean waiting costs under the optimal allocation pattern. 
Remark 4.1. An important observation is that, for the optimal pattern allocation, more load is assigned to the queues with relatively high first moment of the service time distribution than under probabilistic allocation. This property was first reported by Agrawala and Tripathi [1]. The explanation of this property is that the effect of regularizing is stronger for the queues with relatively small assignment probabilities. For example, consider a traffic allocation problem with two queues for which the optimal probabilistic assignment fractions are $p_{1}^{*}=\frac{8}{9}$ and $p_{2}^{*}=\frac{1}{9}$. Then the MAP for the first queue would approximately be equal to a Poisson arrival process with arrival intensity $\frac{8}{9} \Lambda$, hence the switch from probabilistic to pattern allocation would not cause great changes in the arrival process at queue 1 . However, for queue 2 , switching from probabilistic to pattern allocation also changes the arrival process at queue 2 from a Poisson $\left(\frac{1}{9} \Lambda\right)$ into an Erlang $(\Lambda, 9)$ arrival process. The switch from probabilistic to pattern allocation has a more regularizing effect on queue 2 than on queue 1 , and hence the decrement of the mean waiting times is larger for queue 2 than for queue 1 .

This example also shows why the Gamma approximation procedure has a better performance than the approximations obtained from probabilistic allocation: the Gamma arrival process better captures the influence of assignment fractions on the degree of regularization.

Remark 4.2. Elaborating on Remark 4.1, we expect that the effect of a transition from probabilistic allocation to pattern allocation will be stronger when the assignment fractions are closer to each other. In that situation all servers will profit from regularization. An interesting conclusion is that for the case of nonidentical service rates, comparing the Gamma approximation procedure with probabilistic patterns, the difference in patterns is in particular pronounced for low system loads. When the load increases, both methods will lead to allocation fractions close to the capacities of the queues, but for low load probabilistic allocation tends to assign many more customers to the faster queue than the Gamma approximation. Another interesting conclusion is that when the number of servers increases, the effect of regularizing becomes stronger. For example, consider the case of $k$ identical servers with service rate 1 and $\Lambda=\rho k, \rho<1$ and all servers receiving the same fraction $1 / k$ of the arrivals. The allocation pattern based on these fractions leads to $k$ Erlang $(\rho k, k)$ arrival processes. Stoyan [25, Example 1.5.1(e)] shows that Erlang $(\rho(k+1), k+1) \leqslant \mathrm{c}$ Erlang $(\rho k, k)$. Hence the value of the objective function decreases when $k$ increases. Note that for $k \rightarrow \infty$ the arrival processes at the queues become deterministic.

We conclude this section with a remark concerning the validity of our optimization procedure. In this remark we also reveal some problems which occur in the second step of the procedure, where allocation frequencies are to be translated into patterns.

Remark 4.3. Assignment fractions $p_{i}$ do not determine a unique allocation pattern. Firstly, as explained in the previous section, the $p_{i}$ 's can be irrational, so in general a finite pattern with corresponding assignment fractions $p_{i}$ for $i=1, \ldots, N$ does not 
exist. And secondly, even if there exist integer numbers $a_{i}$ such that $p_{i}=a_{i} / \sum_{j} a_{j}$ for $i=1, \ldots, N$, the orders in which the queue numbers can be placed in a pattern are numerous.

However, the natural requirement that the arrival processes should be as regular as possible causes a set of allocation fractions to lead to a more or less uniquely determined allocation pattern. Let us now consider the translation of assignment fractions into patterns.

First, $\left(a_{1}, \ldots, a_{N}\right)$ are defined as follows. For all $\varepsilon>0$, there exists an integer $\bar{m}$ such that

$$
\bar{m}=\min \left\{m>N \mid\left\|\left(p_{i} m-\left[p_{i} m\right]\right) /\left[p_{i} m\right]\right\|<\varepsilon, \quad \frac{\left[p_{i} m\right]}{m} \Lambda<\beta_{i}, \text { for all } p_{i}>0\right\}
$$

Let $a_{i}=\left[p_{i} \bar{m}\right], i=1, \ldots, N$. Hence the $a_{i}$ 's are uniquely defined by a chosen $\varepsilon>0$. Note that the value of $\varepsilon$ has a strong influence on the length of the pattern.

Second, in Section 3 we saw that the mean waiting time decreases with increasing regularity of the arrival process; so given numbers $a_{i}, i=1, \ldots, N$, we try to construct an allocation pattern in which the occurrences of the queue numbers are as uniformly distributed as possible. In this way, given $\varepsilon>0$, assignment fractions $p_{i}$ correspond to a more or less uniquely determined allocation pattern.

This does not imply monotonicity of the waiting times as a function of the assignment fractions. For certain values $p_{i}, i=1, \ldots, N$, placing the queue numbers into a pattern in a uniformly distributed way can be rather difficult, whereas after slightly altering the frequencies, a much more regular pattern would arise. This property also has consequences for the value of the objective function. This contrasts with probabilistic allocation where, given the assignment fractions, the model is equivalent to $N$ independent $\mathrm{M} / \mathrm{G} / 1$ queues.

The actual construction of an allocation pattern is an interesting combinatorial problem, for which we present a heuristic in Appendix B. Here the main problem is that the interests of the queues interfere, i.e. we try to make the arrival process as regular as possible for all queues simultaneously. An example of such interference is, with $N=3, a_{1}=1, a_{2}=2, a_{3}=3$. The reader can easily check that there exists no pattern of length 6 in which the arrival process at all three queues is a renewal process.

In general the optimal allocation pattern cannot be determined, hence it is not to be expected that the optimal assignment frequencies are determined by applying the Gamma approximation procedure. However, our numerical experience indicates that this procedure results in a pattern under which the objective function is close to the approximate lower bound for the optimal arrival pattern, this lower bound being the value of the solution of GA1.

\section{Numerical results}

In this section we present some numerical results. We compare various allocation policies and also discuss the quality of the Gamma approximation procedure. We 
show five instances for the case of two servers and three instances for the case of three servers in parallel. For each instance, the objective is to minimize the mean waiting time of a customer. As a function of the load of the total system, we present absolute and relative values of the objective function for various optimized allocation policies and for the solutions of the mathematical programs.

\subsection{Description of numerical instances and presented results}

In Figs. 1-8 we show numerical results for 8 instances. We have considered the problem of minimizing the mean waiting time of an arbitrary customer (taking $f_{i}(P)=p_{i}, C_{i}=1$, in $\left.(2.2), i=1, \ldots, N\right)$. For each instance, we have optimized various allocation policies for system loads $\rho$ that we increased from 0.05 to 0.95 in steps of 0.05 . The system load is defined as $\rho=\Lambda\left(\sum_{i} 1 / \beta_{i}\right)^{-1}$, i.e. the offered traffic to the system divided by the total service capacity of the system.

Figures 1-5 concern the case of two servers, 6-8 the case of three servers. We have considered three types of service time distributions: exponential, Erlang 2 and hyperexponential. For the hyper-exponential distribution, the coefficient of variation is 2 . In Figs. 1-3 and 6-8 the servers are of the same type, but differ in service rates. For Figs. 4 and 5 the servers are not of the same type; in Fig. 4 both servers have identical service rate, in Fig. 5 the rates are different.

For each instance, two figures are presented, one displaying absolute value of the objective function, the other showing this value relative to the value for optimal probabilistic allocation. The abbreviations used in the figures are:

prob: mean waiting times, under the optimal probabilistic allocation,

prop: pattern that is based on the optimal probabilistic pattern,

klbp: pattern obtained via the gamma approximation procedure, using the KLB approximation for the Gamma/G/1 queue,

klbb: approximate lower bound for the mean waiting times under the optimal allocation pattern (see Section 4),

lb: strict lower bound for the mean waiting times under the optimal allocation pattern. This is for the case of exponential servers (see Section 4),

jlw: mean waiting times under the dynamic policy that allocates a customer to the queue with the least waiting time. These are simulation results.

\subsection{Comparing probabilistic and pattern allocation}

In Section 3 we argued that regularizing arrival processes leads to lower mean waiting times. Also, in Conjecture 3.2 we stated that for each $\mathrm{M} / \mathrm{G} / 1$ queue, there exists a MAP/G/1 queue with lower mean waiting times, where the Poisson arrival process has intensity $p \Lambda, p<1$, and the MAP has the same arrival rate and phase intensity $\Lambda$. We concluded that pattern allocation leads to lower mean waiting times than probabilistic allocation. This conclusion is supported by our numerical results. In all cases considered, the allocation pattern based on the optimal probabilistic allocation fractions (curves prop in Figs. 1-8) performs better than the probabilistic 
allocation itself (prob). The relative differences vary from $7 \%$ to $40 \%$ for low loads up to about $40 \%$ for high load, except for the case of nonidentical servers with identical rate, where the difference for low loads is even $50 \%$. Also, the effect of a transition from probabilistic to pattern allocation is stronger for the case of three servers. All observations are illuminated by Remarks 4.1 and 4.2 .

Figures 1-3 and 6-8 suggest that for identical servers the effect of a transition from probabilistic to pattern allocation is stronger when the service time distribution has a smaller coefficient of variation.

The nonsmoothness of the curves (prop) in Figs. 6-8 is caused by the way the patterns were constructed in our numerical experiments. Due to pattern length limitations, imposed by computer capacity, some inaccuracies occur. The assigned fractions in the pattern are for some values of $\rho$ closer to the optimal probabilistic allocation than for others. It is interesting to see that when the deviation results in relatively speaking - more (less) load at a slower server, this decreases (increases) the value of the objective function. In Remark 4.1 this observation is explained. The effect is most pronounced for $\rho=0.1$, where the constructed allocation pattern actually assigns no customers to the slowest server.

\subsection{Comparing the Gamma approximation procedure with pattern allocation based on optimal probabilistic allocation}

In Section 4 we concluded that the Gamma approximation procedure would lead to better allocation patterns than probabilistic allocation because the Gamma arrival process better captures the behaviour of the MAP than the Poisson arrival process. This conclusion is supported by the numerical results. The difference between objective functions for Gamma approximation based patterns (klbp) and probabilistically based patterns (prop) is larger for lower loads than for higher loads. The difference ranges from $0 \%$ to $45 \%$.

\subsection{Optimal pattern allocation}

Finally, we turn to the questions (i) how good is pattern allocation compared to the best policy, and (ii) how close is the pattern obtained with the Gamma approximation procedure to the optimal allocation pattern?

Concerning (i), it is very hard to determine the optimal - probably a dynamic - allocation policy. Hence we have considered a dynamic policy which is expected to perform better than most policies, and considerably better than the static policies. This dynamic policy operates under complete knowledge of the system at the moment of arrival and sends each customer to the queue with smallest waiting time. Our claim that this is a nearly optimal allocation policy, is based on the fact that this policy uses all information available at moments of arrival and seems to use this information in a very sensible way. In the figures one can see that this dynamic policy ( jlw) performs from $40 \%$ up to about $95 \%$ better than probabilistic allocation. The difference with the optimal Gamma approximated pattern ranges from $20 \%$ up to $45 \%$. 
Concerning question (ii), we know that it is very hard to determine the optimal allocation pattern. However, in an indirect way we are able to make a statement about the quality of the Gamma approximation procedure. In Section 4 we stated that waiting times in a MAP/G/1 queue are bounded from below by the waiting times in the corresponding Gamma/G/1 queue. Numerical experience shows that the approximation of the mean waiting times in the MAP/G/1 queue, using the KLB formula for waiting times in the corresponding Gamma/G/1 queue, is fairly accurate. Hence the value of the objective function for the solution of mathematical program GA1 (klbb) is an approximate lower bound for the optimal allocation pattern.

We also note that, in particular for high loads, this value reasonably accurately approximates the mean waiting times under the allocation pattern that is constructed from the solution of GA1.

So GA1 provides an approximate lower bound for the mean waiting times under the optimal allocation pattern, as well as an accurate approximation of the mean waiting times under the allocation pattern that is based on the solution of GA1. We conclude that the Gamma approximation procedure provides us with a nearly optimal allocation pattern.

Exponential Servers, Absolute Values

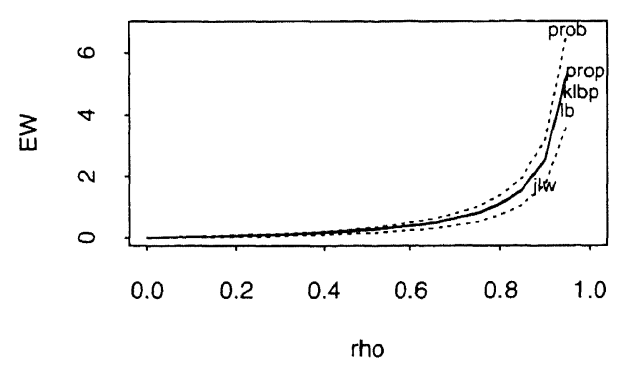

Exponential Servers, Relative Values

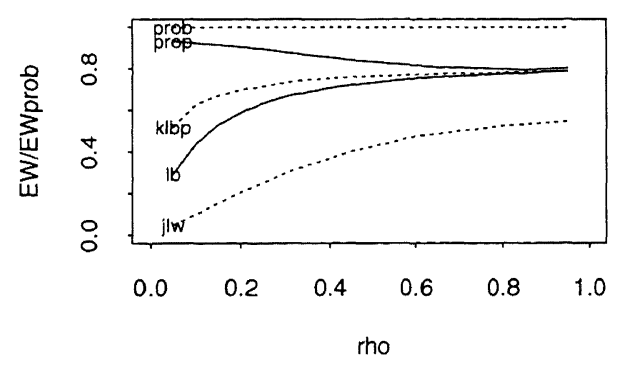

Fig. 1. Two exponential servers, with service rate 1 and 4 , respectively.

Erlang 2 Servers, Absolute Values

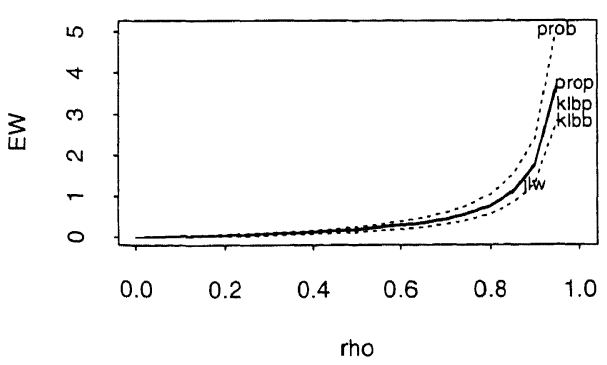

Erlang 2 Servers, Relative Values

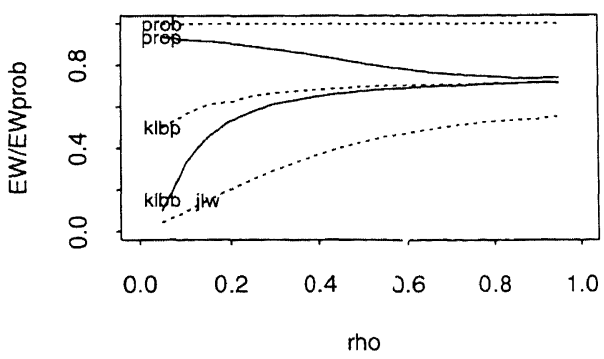

Fig. 2. Two Erlang 2 servers, with service rate 1 and 4, respectively. 
H2 Expo. Servers, Absolute Values

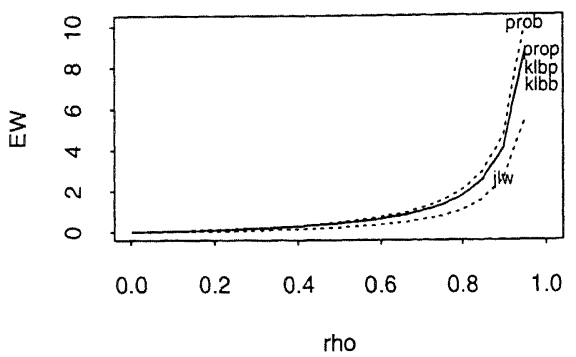

H2 Expo. Servers, Relative Values

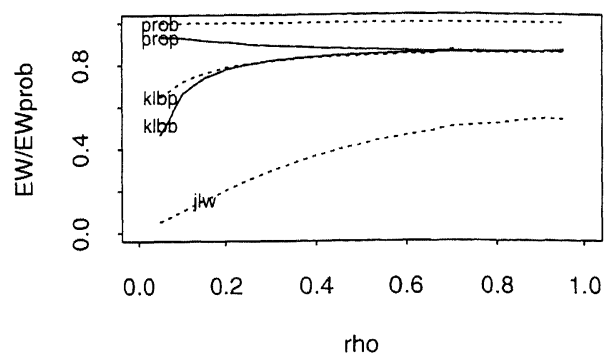

Fig. 3. Two hyper-exponential servers, with service rate 1 and 4 , respectively. The service time of a customer is with probability $q=\frac{1}{3}$ exponentially distributed with parameter $\frac{1}{2} \mu$, and has with probability $1-q=\frac{2}{3}$ an exponential distribution with parameter $2 \mu$. In this way the service rate is $\mu$ and the coefficient of variation is 2 .

Mixed Type Servers, Absolute Values

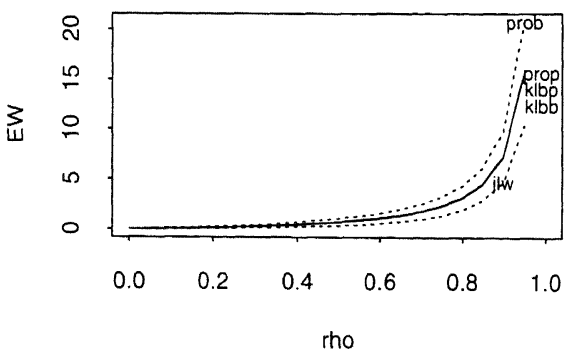

Mixed Type Servers, Relative Values

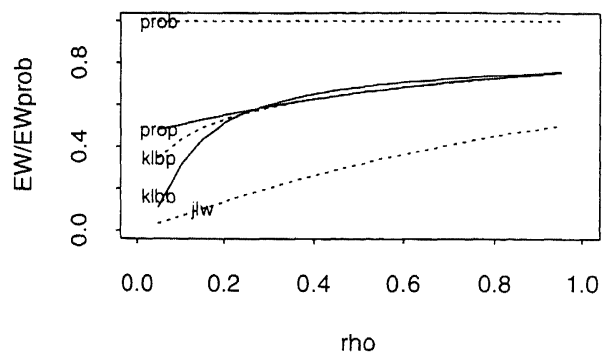

Fig. 4. Two servers with service rate 1 . The first server has Erlang 2 distributed service times, the second server has hyper-exponentially distributed service times as described by Fig. 3.

Mixed Type Servers, Absolute Values

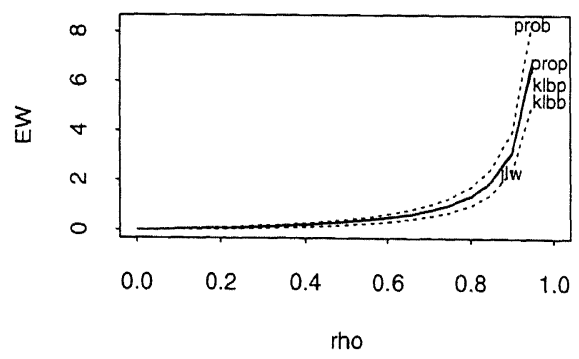

Mixed Type Servers, Relative Values

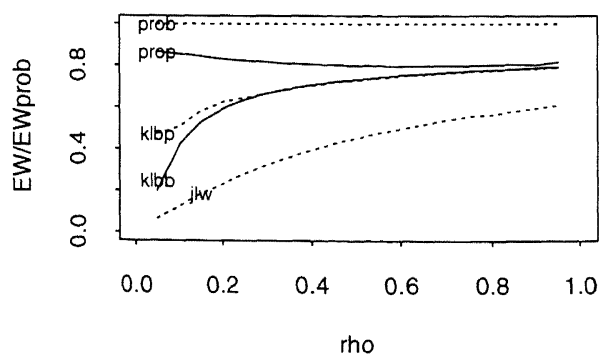

Fig. 5. Two servers. The first server has Erlang 2 distributed service times with rate 1 . The second server has hyper-exponentially distributed service time as described by Fig. 3. 
Exponential Servers, Absolute Values

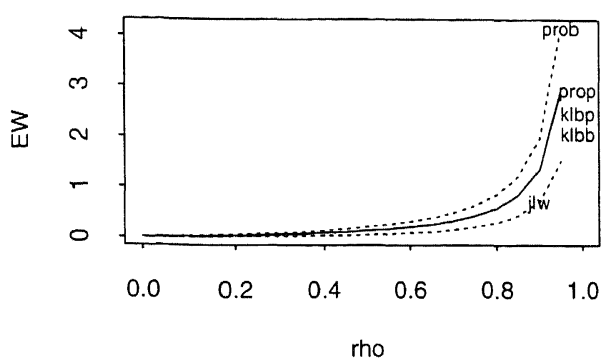

Exponential Servers, Relative Values

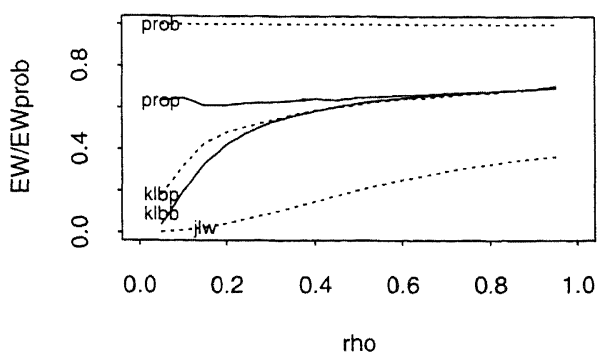

Fig. 6. Three exponential servers, with service rates 1,4 and 7 , respectively.

Erlang 2 Servers, Absolute Values

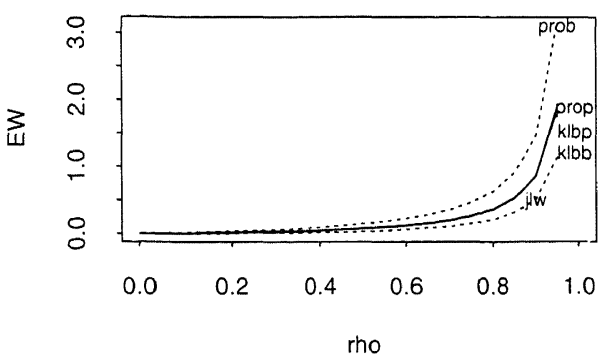

Erlang 2 Servers, Relative Values

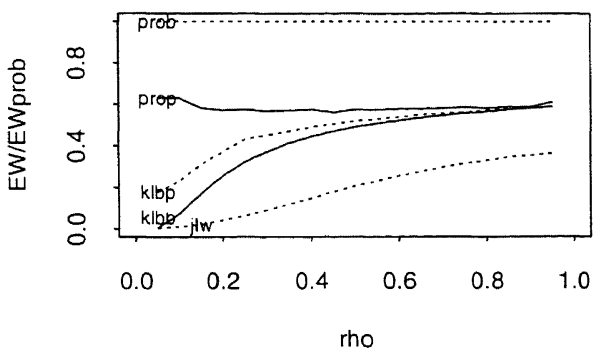

Fig. 7. Three Erlang 2 servers, with service rates 1,4 and 7, respectively.

H2 Expo. Servers, Absolute Values

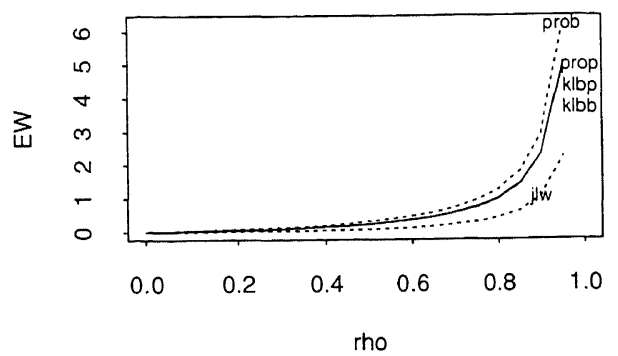

H2 Expo. Servers, Relative Values

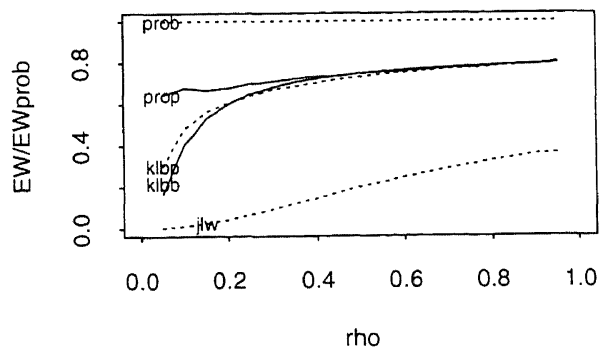

Fig. 8. Three hyper-exponential servers, with service rates 1,4 and 7 , respectively. Service time distributions as described by Fig. 3. 


\section{Extensions of the traffic allocation problem}

In this section we briefly discuss the traffic allocation problem for three extensions of the model that was discussed in the previous sections. For all extensions, the traffic allocation problem can be approached in a similar way as the original problem.

First we discuss the case of general arrival processes. The second model considers the situation in which one has to allocate a Poisson arrival stream to $N$ queues, where each queue already receives a Poisson arrival stream. This problem is known as the traffic allocation problem with dedicated arrival streams. The third model under consideration is the allocation problem with multiple server stations.

In Section 2 we argued that regularizing arrival streams tends to reduce the mean waiting times. Based on similar intuitive arguments, we now make the same conjecture for the extended allocation problems, realizing that proving the same statements for the extended models can only be harder than for the original allocation problem.

According to this assumption, the customers are allocated using an allocation pattern rather than assigning them to the queues probabilistically.

\subsection{Allocation for the case of general arrival processes}

In many traffic allocation situations, the arrival process will not be a Poisson process. We believe that our approach can be extended for such situations. The first step would be to approximate the arrival process by a Gamma process, fitting the parameters $\hat{A}$ and $c_{\mathrm{a}}^{2}$, the arrival rate and squared coefficient of variation, respectively. Hence the interarrival time has the $\operatorname{LST}(\Lambda /(\Lambda+\omega))^{1 / c_{\mathrm{a}}^{2}}$, with $\Lambda=\hat{\Lambda} / c_{\mathrm{a}}^{2}$. Subsequently, applying pattern allocation would result in sending one out of each $y_{i}$ customers to queue $i$. Of course $y_{i}$ need not be integral. However, in an ideal pattern allocation, the interarrival time at queue $i$ will have an LST $(\Lambda /(\Lambda+\omega))^{y_{i} / c_{a}^{2}}$, and hence will be Gamma distributed. We thus can again approximate the optimal assignment frequencies by solving a mathematical programming problem of the form of optimization problem GA1, with constraint $\sum_{i=1}^{N} \alpha_{i}=1$ replaced by $\sum_{i=1}^{N} \alpha_{i}=c_{\mathbf{a}}^{2}$.

\subsection{The allocation problem with dedicated arrival streams}

A Poisson arrival stream with intensity $\Lambda$ has to be allocated to $N$ queues queue $i$, each queue already receiving a Poisson arrival stream with intensity $\lambda_{i}^{\mathrm{d}} \geqslant 0, i=1, \ldots, N$. Note that the original problem returns if $\lambda_{i}^{\mathrm{d}}=0$ for $i=1, \ldots, N$. For the allocation problem with dedicated arrival streams, for the case of exponential servers, $\mathrm{Ni}$ and Hwang [20] optimize the probabilistic allocation policy with the mean sojourn time of a customer as objective fuction.

The benefit of using pattern allocation is less substantial than in the original allocation problem without dedicated arrivals, because allocated arrivals from the additional Poisson arrival stream (forming a MAP) join in with the arrivals from the dedicated Poisson $\left(\lambda_{i}^{d}\right)$ arrival stream. So the arrival processes at the queues are not as 
regular as the MAP in the original problem, they are the sum of such a MAP and a Poisson arrival process. Although the sum of two MAPs is also a MAP, and the Poisson process is just a special MAP, the arrival processes at the queues are hard to approximate by any GI arrival process, in particular the Gamma arrival process.

As a result, we try to approximate the optimal assignment fractions for the allocation pattern with probabilistic allocation or with the Gamma optimization procedure, depending on the ratio between the sum of the dedicated arrival rates and the extra arrival rate. For example: if the sum of the dedicated arrival rates is large compared to the rate of the extra arrival stream, then the resulting arrival processes will resemble a Poisson process more than a MAP, hence it makes more sense to use the assignment fractions from probabilistic allocation for the allocation pattern.

Below the MPP is formulated for the probabilistic allocation policy; for the Gamma optimization procedure the formulation is quite similar.

DA1:

$$
\begin{aligned}
& \min \sum_{i=1}^{N} f_{i} C_{i} \frac{\left(\lambda_{i}+\lambda_{i}^{\mathrm{d}}\right) \beta_{i}^{(2)}}{2\left(1-\left(\lambda_{i}+\lambda_{i}^{\mathrm{d}}\right) \beta_{i}\right)} \\
& \text { s.t. } \sum_{i=1}^{N} \lambda_{i}=\Lambda, \\
& \lambda_{i}+\lambda_{i}^{\mathrm{d}}<\frac{1}{\beta_{i}}, \quad i=1, \ldots, N, \\
& \lambda_{i} \geqslant 0, \quad i=1, \ldots, N .
\end{aligned}
$$

The structure of DA1 is similar to the earlier presented MPPs PA1 and GA1. Again the solution of DA1 can easily be determined using the allocation algorithm in Combé and Boxma [10].

Note that under probabilistic allocation, the original probabilistic allocation problem reappears with the additional constraints that the arrival rate at queue $i$ should be at least $\lambda_{i}^{\mathrm{d}}, i=1, \ldots, N$.

We finish this discussion of load balancing with dedicated arrival streams by mentioning two references on this topic.

Bonomi and Kumar [5] discuss an adaptive probabilistic allocation policy for the case of exponential and the case of identical servers with objective function the mean sojourn time of a customer. They consider the situation where not all system parameters are known, or where some of the parameters may change from time to time. Their main concern is the speed of convergence of the allocation policy towards the optimal assignment probabilities.

Ross and Yao [23] consider the following $N$-server model. At server $i$ a set $S_{i}$ of customer types arrives according to Poisson processes with arrival intensities $\lambda_{i j}, j \in S_{i}$, $i=1, \ldots, N$. The $j$ th arrival stream at server $i$ has service time distribution $B_{i j}(\cdot), j \in S_{i}$, $i=1, \ldots, N$. Furthermore, each server generates additional customers, according to a Poisson process, which may be routed to one of the other servers. The service time of 
such a customer at server $i$ has distribution $B_{i}(\cdot)$. The aim in [23] is to find the probabilistic allocation policy that minimizes the sum of the mean sojourn time and some rerouting delay of a customer from the additional arrival process, under the constraints that the mean sojourn time of the $j$ th dedicated customer stream at server $i$ is less than or equal to $\alpha_{i j}, j \in S_{i}, i=1, \ldots, N$. Ross and Yao [23] allow local priority scheduling of customer types, which also involves the additional customers. The essential problem is to derive an expression for $E \boldsymbol{R}_{i}$, the mean sojourn time at server $i$ of an additional customer when local priority scheduling of customers is allowed. Using matroid theory, Ross and Yao [23] prove that $x_{i} E \boldsymbol{R}_{i}$ is a convex function in $x_{i}$, where $x_{i}$ denotes the additional load assigned to server $i$. The remaining problem, determining the optimal assignment vector $x^{*}=\left(x_{1}^{*}, \ldots, x_{N}^{*}\right)$, proceeds in a way that is similar to solving a common RAP.

It might be interesting to use the resulting assignment vector for determining a good pattern allocation.

\subsection{Allocation for the case of multiple server queues}

In this model, a Poisson arrival stream with intensity $\Lambda$ has to be allocated over $N$ multiple server queues, where the number of servers at queue $i$ is $s_{i}, i=1, \ldots, N$.

Except for a few special cases, no explicit expressions for the mean waiting times in $\mathrm{GI} / \mathrm{G} / \mathrm{s}$ or $\mathrm{MAP} / \mathrm{G} / \mathrm{s}$ queueing systems are available. Hence in this model, an optimal allocation cannot be determined analytically, for both probabilistic assignment and pattern allocation.

We again assume that regularizing the arrival streams decreases mean waiting times, and again we expect to obtain better allocation fractions using the Gamma $/ \mathrm{G} / s_{i}$ approximation for the $\mathrm{MAP} / \mathrm{G} / s_{i}$ queue than when using the $\mathrm{M} / \mathrm{G} / s_{i}$ approximation.

Using this assumption, the path towards an allocation pattern is reasonably straightforward; by choosing a suitable strictly convex approximation for the mean waiting times in a Gamma/G/s queue one can formulate a MPP which possesses all the required properties for applying the algorithm in [10]. Bitran and Dasu [3] and Buzacott and Shanthikumar [7] mention several approximations for the mean waiting time in the $\mathrm{GI} / \mathrm{G} / \mathrm{s}$ queue.

\section{Acknowledgment}

The authors are indebted to Professor J.W. Cohen for carefully reading the manuscript, to S.C. Borst for valuable suggestions concerning the algorithm for constructing an allocation pattern, and to G.M. Koole for interesting discussions.

\section{Appendix A. Waiting times in the MAP/G/1 queue}

In this appendix we present some results on the waiting times of customers in the $\mathrm{MAP} / \mathrm{G} / 1$ queue, and apply them to the typical MAP/G/1 queue which arises under 
pattern allocation. The papers by Lucantoni [17], Ramaswami [22] and the book of Neuts [19] discuss in great detail all aspects of the MAP and the MAP/G/1 queue as parts of a more general framework. In [17] analytical results are obtained for queue lengths, busy period lengths, and waiting times in the MAP/G/1 queue. Lucantoni [17] also provides algorithms to obtain explicit results for distributions and moments of distributions.

The MAP is defined by a continuous-time Markov process $\{J(t), t \geqslant 0\}$ with a finite state space $E=\{1, \ldots, M\}$, where arrivals can occur at transition epochs. $E$ represents the set of phases of the arrival process.

The Markov process has generator $D$, which can be decomposed in two $M \times M$ matrices, $D_{0}$ and $D_{1}$. Let $\lambda_{i j}$ be the transition rate from state $i$ to $j, i \neq j \in E$. Then $\lambda_{i}=$ $\sum_{j \in E, j \neq i} \lambda_{i j}$ is the total transition rate out of state $i$. Let $q_{i j}$ denote the probability that given a transition from state $i$ to $j$ an arrival occurs. Then $D=D_{0}+D_{1}$ where

$$
\begin{aligned}
& \left(D_{0}\right)_{i j}= \begin{cases}-\lambda_{i}, & i, j \in E, \quad i=j, \\
\lambda_{i j}\left(1-q_{i j}\right), & i, j \in E, i \neq j,\end{cases} \\
& \left(D_{1}\right)_{i j}= \begin{cases}0, & i, j \in E, \quad i=j, \\
\lambda_{i j} q_{i j}, & i, j \in E, \quad i \neq j .\end{cases}
\end{aligned}
$$

$D_{0}\left(D_{1}\right)$ represents transitions in the Markov process $\{J(t), t \geqslant 0\}$ without (with) arrivals of customers.

The fundamental arrival rate for the MAP is defined as

$$
\lambda^{\prime}=\pi D_{1} e,
$$

in which $\pi$ is the stationary probability row vector of the Markov process with generator $D$ and $e$ the $M$-dimensional unit column vector.

For the MAP/G/1 queue, let the service time $B$ have an arbitrary distribution $B(\cdot)$ with first and second moment $\beta$ and $\beta^{(2)}$, respectively, and let $\beta(\cdot)$ be the Laplace-Stieltjes transform of $\boldsymbol{B}$. Neuts [19] shows that the MAP/G/1 queue is stable if $\lambda^{\prime} \beta<1$.

Let $W_{v}(\cdot)=\left\{W_{1}(\cdot), \ldots, W_{M}(\cdot)\right\}$, where $W_{j}(x)$ is the joint probability that at an arbitrary time the arrival process is in phase $j$ and the amount of work at the server is at most $x . W_{v}$ is the row vector of the virtual waiting times.

A basic result for the MAP/G/1 queue is the Laplace-Stieltjes transform of $\boldsymbol{W}_{\mathrm{v}}$ (which is the matrix equivalent of the Pollaczek-Khintchine formula for the ordinary $M / G / 1$ queue):

$$
\tilde{W}_{\mathrm{v}}(s)=s\left(1-\lambda^{\prime} \beta\right) g\left[s I+D_{0}+\beta(s) D_{1}\right]^{-1}, \quad s \geqslant 0 .
$$

Here $g$ is the invariant probability vector of a matrix $G=\left(G_{i j}\right), G_{i j}$ is the probability that at the end of a busy period the arrival process is in phase $j$, given that it was in state $i$ at the beginning of that busy period. $G$ can be computed using the following 
matrix functional equation:

$$
G=\int_{x=0}^{\infty} \mathrm{e}^{\left(D_{0}+D_{1} G\right) x} \mathrm{~d} B(x)
$$

The functional equation (A.2) is obtained by an extension of the branching argument for the $M / G / 1$ busy period (cf. [9, p. 249]).

From (A.1), for the specific MAP/G/1 queue which arises under pattern allocation, one obtains $E W_{\mathrm{v}}$, the vector of mean virtual waiting times:

$$
E \boldsymbol{W}_{\mathrm{v}}=\left(E \boldsymbol{W}_{\mathrm{v}}\right) e \pi+\pi-\left(\left(1-\lambda^{\prime} \beta\right) g+\beta \pi D_{1}\right)(e \pi+D)^{-1},
$$

in which $\left(E \boldsymbol{W}_{\mathrm{v}}\right) e$ is given by

$$
\left(E W_{v}\right) e=\frac{1}{2\left(1-\lambda^{\prime} \beta\right)}\left[2\left(\lambda^{\prime} \beta-\left(\left(1-\lambda^{\prime} \beta\right) g+\pi \beta D_{1}\right)(e \pi+D)^{-1} \beta D_{1} e\right)+\lambda^{\prime} \beta^{(2)}\right] .
$$

Using (A.3), the PASTA property, and remarking that for the typical MAP that we study $p_{i j}=1$ if $j=(i \bmod M)+1$, we obtain $E W_{\mathrm{MAP} / \mathrm{G} / 1}$, the mean waiting time of a customer in the $\mathrm{MAP} / \mathrm{G} / 1$ queue:

$$
E W_{\mathrm{MAP} / \mathrm{G} / 1}=E \boldsymbol{W}_{\mathrm{v}} \frac{D_{1} e}{\Lambda} .
$$

\section{Appendix B. Constructing the allocation pattern}

In this appendix we discuss the problem of constructing an allocation pattern from a given set $\left(p_{1}, \ldots, p_{N}\right)$ of allocation fractions. First of all, these frequencies are translated into the vector $\left(a_{1}, \ldots, a_{N}\right)$, in which $a_{i}$ is the number of occurrences of index $i$ in the pattern. The integers $a_{i}$ are computed by defining

$$
\bar{m}=\min \left\{m>N \mid\left\|\left(p_{i} m-\left[p_{i} m\right]\right) /\left[p_{i} m\right]\right\|<\varepsilon, \frac{\left[p_{i} m\right]}{m} \Lambda<\beta_{i}, \text { for all } p_{i}>0\right\}
$$

and taking $a_{i}=\left[p_{i} \bar{m}\right], i=1, \ldots, N$. Note that the choice of $\varepsilon$ has a strong influence on the length of the pattern. After this translation there remains the problem of determining an allocation pattern, such that the number of arriving customers at the routing point between two consecutive allocations to queue $i$ is as constant as possible. Moreover, one has to achieve this for all queues simultaneously. In various optimization problems this combinatorial cyclic scheduling problem has been encountered. Itai and Rosberg [14] suggest the so-called Golden Ratio method for a cyclic scheduling problem that arises in the access control for a multi-access channel. Boxma et al. [6] study a polling model in which a server visits the queues according to a polling table. For this more or less dual problem of the traffic allocation problem they follow an optimization procedure which is similar to our approach for the traffic 
allocation problem. First good visit frequencies are computed for a polling model in which the server chooses his next queue probabilistically, subsequently a polling table based on these frequencies is constructed, using the Golden Ratio method.

The combinatorial complexity of the cyclic scheduling problem is yet undetermined. However, it seems to be a hard problem; it can be translated to known NP-hard problems, although with special structures, but those special structures do not seem to reduce the problem to a polynomially solvable one.

In this appendix a heuristic based on the paper by Hajek [12] on extremal splittings of point processes is presented. This heuristic is an alternative for the Golden Ratio policy as described in [14].

First, some notation and a mathematical criterion for optimality are introduced. A pattern $S$ is defined by $S=\left\{s_{1}, \ldots, s_{k}\right\}$ in which $k=|S|$ is the length of $S$. Let $S_{0}$ be the class of patterns of length $M=\sum_{i}\left[f_{i} \bar{m}\right]$ in which index $i$ occurs exactly $a_{i}$ times. In the rest of this appendix we assume that $a_{1} \geqslant a_{2} \geqslant \cdots \geqslant a_{N}$ and we set $N$ equal to the number of queues with $a_{i}>0$. Under the allocation pattern $S \in S_{0}$, the interarrival times at queue $i$ form a repeated sequence of $a_{i} \operatorname{Erlang}\left(\Lambda, d_{i_{j}}(S)\right)$ distributed variables, $j=1, \ldots, a_{i}$. The "distances" $d_{i_{j}}(S)$ are the numbers of arriving customers at the routing point between two consecutive allocations to queue $i$.

Next, the problem is to determine $S^{*}=\operatorname{argmax}_{S \in S_{0}} V(S)$, in which

$$
V(S)=\left\{\sum_{i} a_{i} \sum_{j=1}^{a_{i}} d_{i_{j}}^{2}(S)\right\}
$$

The objective function $V(\cdot)$ tries to capture the notion of even spreading in the pattern by a kind of second moment function. The weights $a_{i}$ have been chosen such that in the optimal allocation, i.e. $d_{i_{j}}=M / a_{i}, j=1, \ldots, a_{i}, i=1, \ldots, N$, the contributions of the queues to the objective function are all equal. The optimality criterion is quite arbitrary, for example, the weight factors could also have favored the queues with high or those with low frequencies. The same holds for the order in which the indices are included. At the moment it is unclear which objective function is best. However, our numerical experience suggests that slightly altering these factors does not have a substantial influence on the value of the objective function.

The algorithm: The algorithm consists of two phases. In Phase 1 a basic pattern is created with a method derived from [12]. In Phase 2, this pattern is improved with the use of a local search method.

Phase 1: A basic pattern is constructed in an iterative way, starting with an empty pattern and consecutively inserting the indices of the queues into the pattern. After step $i$, the algorithm has produced a sub-pattern $S_{i}$, which contains the indices of queues $1, \ldots, i$. The method operates as follows: If in step $i$ in sub-pattern $S_{i-1}$ of length $k$, the index of queue $i$ has to be inserted $a_{i}$ times, then first the distances $d_{i_{j}}$ for the next sub-pattern $S_{i}$ are computed, following [12], by $d_{i j}=\left[j^{\left(k+a_{i}\right) / a_{i}}\right], j=1, \ldots, a_{i}$. In this way, the distances for queue $i$ are regularly placed around their mean $\left(k+a_{i}\right) / a_{i}$. After computing these distances $d_{i,}$, the indices still can be inserted in 
various ways into $S_{i-1}$. To illustrate, if $S_{2}=\{1,1,2\}$ and $a_{3}=1$, then there are three different patterns to choose $S_{3}$ from: $\{3,1,1,2\},\{1,3,1,2\}$ and $\{1,1,3,2\}$. In this example, the insertion can start from three different points in $S_{2}$. In general, there can be $k$ different ways of inserting, creating possible new sub-patterns $S_{i}^{1}, \ldots, S_{i}^{k}$. From these patterns, $S_{i}$ is chosen such that $V\left(S_{i}\right)=\min _{1 \leqslant j \leqslant k} V\left(S_{i}^{j}\right)$.

We see that in the $i$ th step of phase 1 , index $i$ is optimally placed in the sub-pattern. However, this optimality could be ruffled in subsequent iteration steps. Therefore in phase 2 a local search method is applied, trying to restore some of the regularity.

Phase 2: In the local search $S_{N}$ is replaced by $S_{N}^{\prime}(k, l)$ if $V\left(S_{N}^{\prime}(k, l)\right)<V\left(S_{N}\right)$, where $S_{N}^{\prime}(k, l)=S_{N}$ except for entries $k$ and $l$, which in $S_{N}^{\prime}(k, l)$ are interchanged compared to $S_{N}$. This local search is repeated until no further improvements can be made.

Remark B.1. For the first phase also the Golden Ratio method, as described by Itai and Rosberg [14], could have been applied. The local search method does improve the Golden Ratio pattern, but in general the above-described heuristic based on [12] performs better. In the cases that we ran, the latter method provides a pattern $S$ for which the objective function $V(S)$ lies between 0 and 5 percent of the theoretical minimum, whereas Golden Ratio's relative error is in most cases between 2 and 4 times as high.

\section{References}

[1] A.K. Agrawala and S.K. Tripathi, On the optimality of semidynamic routing schemes, Inform. Process Lett. 13 (1981) 20-22.

[2] A.K. Agrawala and S.K. Tripathi, On an exponential server with general cyclic arrivals, Acta Inform. 18 (1982) 319-334

[3] G.R. Bitran and S. Dasu, A review of open queueing networks models of manufacturing systems, Queueing Systems 12 (1992) 95-133.

[4] R.K. Boel and J.H. van Schuppen, Distributed routing for load balancing, Proc. IEEE 77 (1989) 210-221.

[5] F. Bonomi and S. Kumar, Adaptive optimal load balancing in a nonhomogeneous multiserver system with a central job scheduler, IEEE Trans. Comput. C-39 (1990) 1232-1250.

[6] O.J. Boxma, H. Levy and J.A. Weststrate, Efficient visit frequencies for polling tables: minimization of waiting costs, Queueing Systems 9 (1991) 133-162.

[7] J.A. Buzacott and J.G. Shanthikumar, Design of manufacturing systems using queueing models, Queueing Systems 12 (1992) 135-213.

[8] J.P. Buzen and P.P.-S. Chen, Optimal load balancing in memory hierarchies, in: J.L. Rosenfeld, ed., Proc. IFIP 1974 (North-Holland, Amsterdam, 1974) 271-275.

[9] J.W. Cohen, The Single Server Queue (North-Holland, Amsterdam, 2nd ed., 1982).

[10] M.B. Combé and O.J. Boxma, Optimization of static traffic allocation policies, CWI Report BSR9302, 1993.

[11] A. Ephremides, P. Varaiya and J. Walrand, A simple dynamic routing problem, IEEE Trans. Automat. Control AC-25 (1980) 690-693.

[12] B. Hajek, Extremal splittings of point processes, Math. Oper. Res. 10 (1985) 543-556.

[13] T.I. Ibaraki and N. Katoh, Resource Allocation Problems (MIT Press, Cambridge, 1988).

[14] A. Itai and Z. Rosberg, A Golden Ratio control policy for a multiple-access channel, IEEE Trans. Automat. Control AC-29 (1984) 399-419. 
[15] A. Jean-Marie, Load balancing in a system of two queues with resequencing, in: P.J. Courtois and G. Latouche, eds., Proc. Performance'87 (North-Holland, Amsterdam, 1988) 75-88.

[16] W. Krämer and M. Langenbach-Belz, Approximate formulae for the delay in the queueing system GI/G/1, in: Proc. 8th ITC Congr. (Melbourne, 1976) 235.1-235.8.

[17] D.M. Lucantoni, New results on the single server queue with a batch Markovian arrival process, Comm. Statist. Stochastic Models 7 (1991) 1-46.

[18] M.F. Neuts, A versatile Markovian arrival process, J. Appl. Probab. 16 (1979) 764-779.

[19] M.F. Neuts, Structured Stochastic Matrices of $M / G / 1$ Type and their Applications (Dekker, New York, 1989).

[20] L.M. Ni and K. Hwang, Optimal load balancing in a multiple processor system with many job classes, IEEE Trans. Software Engrg. SE-11 (1985) 492-496.

[21] K.K. Ramakrishnan, The design and analysis of resource allocation policies in distributed systems, Ph.D. Thesis, Dept. of Computer Science, Univ. of Maryland, MD, 1983.

[22] V. Ramaswami, The N/G/1 queue and its detailed analysis, Adv. in Appl. Probab. 12 (1980) 222-261.

[23] K.W. Ross and D.D. Yao, Optimal load balancing and scheduling in a distributed computer system. J. $A C M 38$ (1991) 676-690.

[24] J.G. Shanthikumar and J.A. Buzacott, On the approximations to the single server queue, Internat. $J$ Prod. Res. 18 (1980) 761-773.

[25] D. Stoyan, Comparison Methods for Queues and other Stochastic Models (Wiley, New York, 1983); translated and revised version of German original (1977).

[26] C.S. Tang and M. van Vliet, Traffic allocation for manufacturing systems, Tech. Report 9116/A, Econometric Inst., Erasmus Univ., Rotterdam, 1991; European J. Oper. Res., to appear.

[27] Y-T. Wang and R.J.T. Morris, Load sharing in distributed systems, IEEE Trans. Comput. C-34 (1985) 204-217.

[28] T.P. Yum, The design and analysis of a semi-dynamic deterministic routing rule, IEEE Trans. Comm. 29 (1981) $498-504$ 